\title{
ĐỔI MỚI CHỈ TIÊU ĐÁNH GIÁ ĐÔ CHÍNH XÁC ĐO ĐẠC ĐỊA HİNH TRÊN NỀN CÔNG NGHỆ ẢNH KỸ THUẠT SỐ
}

\author{
NGUYẼ̃N TH!̣ BÍCH NGỌC(1), ĐÀO TH!̣ NGA ${ }^{(2)}$ \\ ${ }^{(1)}$ Cục Đo đạc, Bản đồ và Thông tin địa lý Việt Nam \\ ${ }^{(2)}$ Tổng công ty Tài nguyên và Môi trường Việt Nam
}

\section{Tóm tắt:}

Công nghệ thành lập bản đồ và đo đạc thu nhận dữ liệu địa lý trong những năm qua đã có nhiều thay đổi, nhiều công nghệ mới đã được đưa vào sử dụng ở Việt Nam. Trong khi đó, ở một số tiêu chuẩn kỹ thuật, việc đánh giá chất lượng sản phẩm vẫn áp dụng theo các quy định cũ. Bài báo giới thiệu kết quả nghiên cứu trong việc đổi mới chỉ tiêu đánh giá độ chính xác đo đạc địa hình trên nền công nghệ ảnh kỹ thuật số.

\section{1. Đặt vấn đề}

Trong những năm qua quy trình thành lập bản đồ địa hình tại Việt Nam đã có những thay đổi nhất định, đó là việc có thêm một loại sản phẩm là dữ liệu địa lý, độ chính xác dữ liệu được đánh giá và công bố như một thuộc tính trong siêu dữ liệu. Theo đó, cần có các tiêu chuẩn kỹ thuật tương ứng với quy trình, công nghệ áp dụng để thu nhận dữ liệu. Theo đó, việc đổi mới chỉ tiêu đánh giá độ chính xác kết quả đo đạc địa hình theo phương pháp trực tiếp dựa trên các căn cứ khoa học và thực tiễn sau:

Kết quả thực hiện đề tài cấp Bộ: "Nghiên cứu cơ sở khoa học và thực tiễn phục vụ đổi mới chỉ tiêu đánh giá độ chính xác mặt phẳng và độ cao trong đo đạc địa hình ở Việt Nam trên nền công nghệ không ảnh và đo đạc trực tiếp toàn số hiện nay";

Kế thừa Tiêu chuẩn của Hiệp hội Chụp ảnh và Viễn thám Hoa Kỳ (ASPRS-1990) với mức khởi tạo (loại I) là 0.05 feet (trên thực địa), tương ứng với 0.0125 met $(1.25 \mathrm{~cm})$, các mức tiếp theo được phân chia dựa trên mẫu số của các loại tỷ lệ bản đồ địa hình cơ bản;

Tuân thủ Quy chuẩn quốc gia về Chuẩn thông tin địa lý cơ sở, mã số QCVN42:2012/BTNMT về đánh giá chất lượng dữ liệu và ghi nhận siêu dữ liệu, có sự kế thừa các tiêu chuẩn quốc tế (ISO 19131) và các quy định đánh giá độ chính xác trong các Thông tư quy định kỹ thuật về dữ liệu địa lý;

Đổi mới chỉ tiêu đánh giá độ chính xác kết quả đo đạc địa hình theo phương pháp gián tiếp được thiết lập với các thông số kỹ thuật phục vụ xây dựng tiêu chuẩn kỹ thuật đo vẽ địa hình bằng công nghệ ảnh toàn số, thay thế cho các văn bản quy định thành lập bản đồ bằng công nghệ ảnh số (ban hành từ năm 2005).

2. Bộ chỉ tiêu đánh giá độ chính xác đo đạc địa hình trên nền công nghệ đo ảnh kỹ thuật số

Chỉ tiêu về độ chính xác được áp dụng trong các tiêu chuẩn kỹ thuật (TCKT) với các nhóm tiêu chuẩn thành phần sau:

Nhóm tiêu chuẩn đánh giá chất lượng dữ liệu địa lý theo phương pháp trực tiếp;

b) Nhóm tiêu chuẩn đánh giá chất lượng dữ liệu địa lý theo phương pháp gián tiếp.

\subsection{Chỉ tiêu đánh giá độ chính xác theo phương pháp trực tiếp}

Mức chỉ tiêu độ chính xác được xác định phù hợp với khả năng của trang thiết bị công nghệ, nhu cầu khai thác ứng dụng dữ liệu địa lý và điều kiện địa hình. Mức chỉ tiêu loại 1 được áp dụng để ước tính độ chính xác cần đạt được đối với một công nghệ, theo đó là các chỉ tiêu chuẩn kỹ thuật liên quan đến độ chính xác của các hạng mục

Ngày nhận bài: 06/12/2017, ngày chuyển phản biện: 11/12/2017, ngày chấp nhận phản biện: 18/12/2017, ngày chấp nhận đăng: 20/12/2017 
công trình, sản phẩm có liên quan. Chỉ tiêu độ chính xác được áp dụng khi đánh giá và công bố kết quả thực hiện các phép đo độ chính xác của dữ liệu địa lý theo giá trị sai số trung phương của các trị đo trắc địa tính theo đơn vị mét ở thực địa (phương pháp trực tiếp). Trường hợp không thực hiện các phép đo kiểm tra trực tiếp, chỉ tiêu độ chính xác có thể được báo cáo trên cơ sở tuân thủ các tiêu chuẩn, quy chuẩn kỹ thuật đã áp dụng để đạt được mức tương ứng và được cơ quan thẩm quyền công bố (phương pháp gián tiếp).

\section{a) Chỉ tiêu độ chính xác về mặt phẳng}

Thay đổi từ quy định giá trị giới hạn sai số trung phương vị trí điểm chi tiết trên bản đồ tính theo công thức: $m_{p}=\sqrt{ }\left(m_{x}\right)^{2}+\left(m_{y}\right)^{2}$ không vượt quá $0.5 \mathrm{~mm}$ bằng việc thiết lập một dãy các mức giới hạn sai số các thành phần tọa độ $x, y$ của tập dữ liệu địa lý cần được đánh giá bằng tập trị đo có độ chính xác cao hơn. Tỷ lệ bản đồ có ý nghĩa tương tự như việc thể hiện lượng sai số đó trên bản vẽ và là cơ sở để phân định giãn cách giữa các mức và số mức chỉ tiêu độ chính xác về mặt phẳng. Sự thay đổi này đảm bảo tính linh hoạt trong đánh giá độ chính xác của các loại dữ liệu mặt phẳng định dạng vector, raster và cho phép kết luận về mức độ chính xác đạt được cụ thể là bao nhiêu mét. Chỉ tiêu độ chính xác về mặt phẳng được chỉ ra trong bảng 1 . (xem bảng 1)

Thực hiện kiểm tra đô chính xác được đo trực tiếp trên các lớp đổi tượng địa lý hoặc lấy mẫu theo xác xuất. Sau khi tính sai số theo tọa độ $x$ và $y\left(m_{x} m_{y}\right)$, việc kết luận, đánh giá và công bố độ chînh xác đạt được của đối tượng hoặc lớp đối tượng căn cứ vào tiêu chí của từng mức chi tiết, loại độ chính xác hay độ phân giải của dữ liệu (tương ứng với loại tỷ lệ bản đồ). Mức trên $5 \mathrm{~m}$ thường áp dụng đối với bản đồ các tỷ lệ $1: 25.000,1: 50.000$ có thể sử dụng nguồn ảnh vệ tinh độ phân giải $2.5 \mathrm{~m}-1 \mathrm{~m}$.

b) Chỉ tiêu độ chính xác về độ cao

Chỉ tiêu độ chính xác về độ cao được thiết lập độc lập với độ chính xác về mặt phẳng dựa trên kết quả khảo sát khả năng thu nhận dữ liệu độ cao của các công nghệ hiện có. Số mức và giãn cách giữa các mức có tham chiếu đến thể hiện dáng đất trên bản đồ địa hình. Chỉ tiêu độ chính xác về độ cao được chỉ ra trong bảng 2 (xem bảng 2 ). Trong đó độ chính xác mô hình số độ cao được xác định là độ chính xác độ cao của đối tượng mô tả địa hình tham gia vào mô hình TIN. Từ đó, lớp địa hình trên bản đồ có thể được biểu thị bằng các đường đồng mức với khoảng cao đều $0.5 \mathrm{~m}, 1.0 \mathrm{~m}, 2.5 \mathrm{~m}$; Các mức độ chính xác từ $1 \mathrm{~m}$ đến $6.6 \mathrm{~m}$ thường áp dụng cho các khu vực có độ dốc đáng kể (vùng đồi, núi). Đối với mô hình số độ cao (DTM), đối tượng mô tả bề mặt địa hình (sát mặt đất) theo chỉ tiêu $m_{h c t}=1 / 2$ chỉ tiêu độ chính xác của dữ liệu độ cao áp dụng cho các đối tượng đo vẽ trực tiếp trên mô hình lập thể.

\subsection{Chỉ tiêu đánh giá độ chính xác dữ} liệu gián tiếp áp dụng đối với công nghệ đo ảnh hàng không toàn số

Chỉ tiêu đánh giá độ chính xác gián tiếp là các chỉ tiêu kỹ thuật cần được kiểm soát đối với quy trình thực hiện để đảm bảo khai thác khả năng tối đa của công nghệ ở mức chỉ tiêu độ chính xác trực tiếp đã xác định trong quá trình lập thiết kế.

Độ chính xác tuyệt đối của dữ liệu địa lý được đo vẽ, thu nhận bằng công nghệ đo ảnh kỹ thuật số phụ thuộc vào hiệu quả quản lý chất lượng của các quá trình: Thu nhận và xử lý dữ liệu ảnh theo công nghệ GNSS/IMU (sản phẩm là ảnh màu kỹ thuật số kèm theo các nguyên tố định hướng ngoài của ảnh); Xây dựng lưới tam giác ảnh không gian (sản phẩm là các thông số định hướng ảnh được bình sai trong hệ tọa độ, độ cao trắc địa); Nhận dạng và đo vẽ đối tượng mặt phẳng, độ cao theo chỉ tiêu độ chính xác nhất định.

a) Chỉ tiêu kỹ thuật đối với dữ liệu ảnh kỹ thuật số

Liên quan đến độ chính xác bao gồm độ phân giải mặt đất và dữ liệu định hướng 
Bảng 1: Chỉ tiêu độ chính xác tuyệt đối về mặt phẳng

\begin{tabular}{|c|c|c|c|c|c|}
\hline $\begin{array}{c}\text { SSTP } \\
\text { hướng } x, y \\
\mathrm{~m}_{\mathrm{x}}\left(\mathrm{m}_{\mathrm{y}}\right) \\
(\mathrm{m})\end{array}$ & $\begin{array}{c}\text { SSTP } \\
\text { mặt phẳng } \\
\left(\mathrm{m}_{\mathrm{xy}}\right) \\
\left(1.41^{*} \mathrm{~m}_{\mathrm{x}}\right)\end{array}$ & $\begin{array}{l}\text { Độ chính xác } \\
\text { mặt phẳng } \\
\text { mức tin cậy } 95 \% \\
\left(2.45^{*}\left(\mathrm{~m}_{\mathrm{xy}}\right)\right.\end{array}$ & $\begin{array}{c}\text { ĐXC ảnh } \\
\text { trực giao } \\
\text { hướng } X, Y \\
2^{*} m_{x}=2^{*}\left(m_{y}\right)\end{array}$ & $\begin{array}{c}\text { Thể hieên } \\
\text { ở tỷ lệ } \\
\text { Bản đồ }\end{array}$ & $\begin{array}{l}\text { Phân loại } \\
\text { độ chính xác }\end{array}$ \\
\hline 0.0125 & 0.02 & 0.03 & & $1: 50$ & $\mathrm{~T}$ \\
\hline 0.025 & .04 & 0.06 & & & II \\
\hline .0375 & 0.05 & 0.09 & & & III \\
\hline 0.025 & 0.04 & 0.06 & & $1: 100$ & $\mathrm{I}$ \\
\hline 0.0 & 0.07 & 0.12 & & & II \\
\hline 0.075 & 0.11 & 0.18 & & & III \\
\hline 0.05 & 0.07 & 0.12 & & $1: 200$ & $\mathrm{I}$ \\
\hline 0.1 & 0.14 & 0.25 & & & II \\
\hline 0.15 & 0.21 & 0.37 & & & III \\
\hline 0.125 & 0.18 & $\overline{0.31}$ & & $1: 500$ & $\mathrm{I}$ \\
\hline 0.25 & 0.35 & 0.61 & & & II \\
\hline 0.375 & 0.53 & 0.92 & & & III \\
\hline 0.25 & 0.35 & 0.61 & 0.50 & $1: 1.000$ & $\mathrm{I}$ \\
\hline 0.5 & 0.71 & 1.21 & 1.00 & & II \\
\hline 0.75 & 1.06 & 1.84 & 1.50 & & III \\
\hline 0.5 & 0.71 & 1.23 & 1.00 & $1: 2.000$ & $\mathrm{~T}$ \\
\hline 1 & 1.41 & 2.45 & 2.00 & & II \\
\hline 1.5 & 2.12 & 3.68 & 3.00 & & III \\
\hline 1.25 & 1.76 & 3.06 & 2.50 & $1: 5.000$ & $\mathrm{I}$ \\
\hline 2.5 & 3.53 & 6.13 & 5.00 & & II \\
\hline 3.75 & 5.29 & 9.19 & 7.50 & & III \\
\hline 2.5 & 3.53 & 6.13 & 5.00 & $1: 10.000$ & $\mathrm{~T}$ \\
\hline 5 & 7.05 & 12.25 & 10.00 & & II \\
\hline 7.5 & 10.58 & 18.38 & 15.00 & & III \\
\hline 6.25 & 8.81 & 15.31 & 12.50 & $1: 25.000$ & $\mathrm{~T}$ \\
\hline 12.5 & 17.63 & 30.63 & 25.00 & & II \\
\hline 18.75 & 26.44 & 45.94 & 37.50 & & III \\
\hline 12.5 & 17.63 & 30.63 & 25.00 & $1: 50.000$ & $\mathrm{I}$ \\
\hline 25 & 35.25 & 61.25 & 50.00 & & II \\
\hline 37.5 & 52.88 & 91.88 & 75.00 & & III \\
\hline
\end{tabular}

ngoài cuả ảnh. Quan hệ giữa tỷ lệ bản đồ cần thành lập và tỷ lệ bay chụp ảnh trong các quy định hiện hành sẽ được thay thế bằng quan hệ giữa chỉ tiêu độ chính xác và độ phân giải ảnh kỹ thuật số sau đây:

$$
\mathrm{GSD}=\mathrm{H} / \mathrm{f} \times \mathrm{S}
$$

Trong đó: $H$ là độ cao bay chụp so với mặt đất; $f$ là chiều dài tiêu cự ; $S$ kích thước pixel.

* Đối với trường hợp thiết kế độ phân giải thỏa mãn yêu cầu độ chính xác độ cao, độ cao bay chụp $(\mathrm{H})$ trong công thức trên được ước tính theo công thức: $\mathrm{H}=\mathrm{m}_{\mathrm{h}} \frac{\mathrm{b}}{\mathrm{m} \Delta \mathrm{p}}$ (2.2) $\mathrm{m}_{\mathrm{h}}$ : Chỉ tiêu độ chính xác độ cao cần đạt được.

b: đường đáy chụp ảnh (phụ thuộc kích thước CCD, độ phủ dọc).

$\mathrm{m}_{\Delta \mathrm{p}}$ : Sai số trung phương xác định thị sai ngang trong ước tính độ cao bay.

* Đối với trường hợp chỉ cần đáp ứng tiêu chí về độ chính xác mặt phẳng, độ phân giải mặt đất phụ thuộc vào tiêu chí về độ chính xác mặt phẳng $\mathrm{m}_{\mathrm{xy}}$ cần đạt được và 
Bảng 2: Chỉ tiêu độ chính xác tuyệt đối về độ cao

\begin{tabular}{|c|c|c|c|c|c|c|c|c|}
\hline \multirow{2}{*}{$\begin{array}{l}\text { Mức chỉ } \\
\text { tiêu độ } \\
\text { chính xác } \\
\text { độ cao }\end{array}$} & \multirow{2}{*}{\multicolumn{2}{|c|}{$\begin{array}{l}\text { Sai số trung phương } \\
\text { độ cao địa hình rõ ràng } \\
\text { không thực phủ }\end{array}$}} & \multicolumn{4}{|c|}{ Độ chính xác độ cao mức tin cậy 95\% } & \multirow{2}{*}{$\begin{array}{l}\text { Tương ứng } \\
\text { với KCĐ } \\
\text { cơ bản }\end{array}$} & \multirow{2}{*}{$\begin{array}{l}\text { Phù hợp } \\
\text { với độ } \\
\text { dốc địa } \\
\text { hình }\end{array}$} \\
\hline & & & \multicolumn{2}{|c|}{$\begin{array}{c}\text { Địa hình rõ ràng, không } \\
\text { thực phủ }\end{array}$} & \multicolumn{2}{|c|}{$\begin{array}{c}\text { Địa hình khó khăn, } \\
\text { thực phủ }\end{array}$} & & \\
\hline$m_{\text {hct }}$ & \multicolumn{2}{|c|}{$m_{\text {hct }}$} & \multicolumn{2}{|c|}{$\left(m_{\text {hct }}{ }^{*} 1.96\right)$} & \multicolumn{2}{|c|}{$\left(m_{\text {hct }}{ }^{*} 3\right)$} & $m_{\text {hgh }}$ & \\
\hline$(\mathrm{cm})$ & $(\mathrm{cm})$ & $(\mathrm{cm})$ & (cm) & (m) & $(\mathrm{cm})$ & $(\mathrm{m})$ & (m) & \\
\hline 1 & 1 & & 1.96 & & 3 & & - & \\
\hline 2.5 & 2.5 & & 4.9 & & 7.5 & & - & \\
\hline 5 & 5 & & 9.8 & & 15 & & - & \\
\hline 10 & 10 & & 19.6 & & 30 & & - & \\
\hline 12.5 & & 0.13 & & 0.25 & & 0.4 & 0.5 & $0^{\circ}-2^{\circ}$ \\
\hline 2.0 & & 0.25 & & 0.9 & & 0.8 & 1.0 & $0^{\circ}-2^{\circ}$ \\
\hline 33.3 & & 0.33 & & 0.65 & & 1.0 & - & $0^{\circ}-2^{\circ}$ \\
\hline 40.0 & & 0.40 & & 0.78 & & 1.2 & - & $0^{\circ}-2^{\circ}$ \\
\hline 50.0 & & 0.50 & & 0.98 & & 1.5 & - & $2^{\circ}-5^{\circ}$ \\
\hline 66.6 & & 0.67 & & 1.31 & & 2.0 & - & $2^{\circ}-5^{\circ}$ \\
\hline 83.0 & & 0.83 & & 1.63 & & 2.5 & 2.5 & $2^{\circ}-5^{\circ}$ \\
\hline 100.0 & & 1.00 & & 1.96 & & 3.0 & & $2^{\circ}-5^{\circ}$ \\
\hline 166.0 & & 1.66 & & 3.25 & & 5.0 & 5.0 & $6^{\circ}-15^{\circ}$ \\
\hline 333.0 & & 3.33 & & 6.53 & & 10.0 & 10.0 & $6^{\circ}-15^{\circ}$ \\
\hline 666.0 & & 6.66 & & 13.05 & & 20.0 & 20.0 & $15^{\circ}-25^{\circ}$ \\
\hline
\end{tabular}

Ghi chú bảng 1 và bảng :

*Các mức độ chính xác từ $10 \mathrm{~cm}$ trở lên được áp dụng đối với các trường hợp yêu cầu đo độ cao với độ chính xác rất cao, thường là các điểm khống chế có dấu mốc, điểm kiểm tra để đánh giá độ chính xác cho các công nghệ có độ chính xác thấp hơn.

${ }^{*}$ Các mức độ chính xác $20 \mathrm{~cm}-100 \mathrm{~cm}$ (dưới $1 \mathrm{~m}$ ) áp dụng đối với khu vực địa hình khá bằng phẳng nhưng yêu cầu phân tích dữ liệu không gian với độ chính xác cao, phù hợp với công nghệ đo trực tiếp, quét Lidar.

ước tính theo công thức: $m_{x y}=G S D / k$ (hệ số $k$ phụ thuộc vào điều kiện cho phép của trang thiết bị, tính kinh tế và không nhỏ hơn 3). Đối với hệ thống chụp ảnh Vexcel Ultracam XP w/a, tương ứng với độ phân giải mặt đất xác định theo 2.1 và 2.2 , với k>=3, chỉ tiêu độ chính xác tăng dày mặt phẳng được thể hiện ở bảng 4 (xem bảng 4); Chỉ tiêu độ chính xác tăng dày cả mặt phẳng và độ cao được tính toán với sai số đo thị sai ngang lấy bằng 1 pixel (tương đương $\left.\mathrm{m}_{\Delta \mathrm{p}}=6 \mu \mathrm{m}\right)$ được thể hiện trong bảng 5 (xem bảng 5)

b) Chỉ tiêu độ chính xác bình sai lưới tam giác ảnh không gian (TGAKG)

Theo quy định quản lý chất lượng dữ liệu địa lý tại quy chuẩn QCVN 42, trong số các phương pháp đánh giá độ chính xác gián tiếp, chỉ tiêu độ chính xác tăng dày rất có ý nghĩa trong việc loại bỏ sai số hệ thống đối với tập trị đo tọa độ, độ cao của đối tượng địa lý.

Với chất lượng tọa độ tâm chụp được xác định bằng công nghệ GNSS/IMU, khối tam giác ảnh không gian đã được kết nối tương đối khá chặt và sai số biến dạng lưới đã được hạn chế tới mức thấp nhất. Vai trò của khống chế mặt đất chủ yếu là xử lý các sai số hệ thống trong bài toán bình sai lưới TGAKG về hệ tọa độ, độ cao quốc gia.

Đối với ảnh kỹ thuật số dạng chụp khung, kích thước mảng CCD giới hạn, ví dụ (103.9 x 67.8) mm đối với Vecxel, các quy định về giãn cách điểm khống chế theo số đáy ảnh như trong các quy định thành lập bản đồ bằng công nghệ ảnh số (được ban hành năm 2005) đã không còn phù hợp. Một số kết quả nghiên cứu khoa học về công nghệ 
ảnh toàn số đã đề xuất phương án thiết kế mật độ điểm khống chế mặt đất theo khoảng cách tương đối giữa các điểm khống chế hoặc mật độ điểm trên diện tích khu bay tùy thuộc vào độ phân giải mặt đất của ảnh và yêu cầu độ chính xác đo vẽ địa hình. Sau đây là một số khuyến cáo được rút ra từ các nghiên cứu thử nghiệm trong và ngoài nước:

- Trường hợp chỉ tăng dày mặt phẳng, với 04 điểm khống chế mặt đất tại 4 góc khu bay có thể đủ điều kiện để bình sai khối ảnh về hệ tọa độ trắc địa trong trường hợp vị trí điểm khống chế đạt yêu cầu.

- Trường hợp sử dụng ảnh độ phân giải cao (GSD $=12-15 \mathrm{~cm}$ ) để tăng dày cho đo vẽ độ cao độ chính xác $0,3-0.5 \mathrm{~m}$, khu vực địa hình ổn định, rõ rệt có thể bố trí điểm khống chế với giãn cách trong khoảng $3,5-5 \mathrm{~km}$. Trường hợp đo vẽ độ cao độ chính xác $0.7 \mathrm{~m}$ giãn cách điểm có thể tăng lên đến $7 \mathrm{~km}$, trường hợp chất lượng ảnh chụp và thông số định hướng tốt, nhiều khả năng cho phép chọn điểm khống chế ảnh đạt chuẩn, giãn cách đến $10 \mathrm{~km}$ vẫn có thể đạt tới độ chính xác này. Với các chỉ tiêu độ chính xác thấp hơn $(0.85 \mathrm{~m}-1.0 \mathrm{~m})$ điểm khống chế mặt đất được bố trí rải theo mật độ khoảng từ 40-50 km²/điểm. Trong trường hợp chất lượng khống chế đạt chuẩn mật độ này có thể tăng lên đến $80 \mathrm{~km}^{2}$.

- Trường hợp sử dụng ảnh độ phân giải trung bình $(G S D=40 \mathrm{~cm})$ về lý thuyết có thể thiết kế phương án bay chụp ảnh số để đo vẽ độ chính xác độ cao đến $1.0 \mathrm{~m}$. Trên thực tế, sau khi đo vẽ mô tả địa hình, độ chính xác độ cao tại khu vực rõ ràng, không thực phủ có thể đạt được tới $0.5 \mathrm{~m}$. Đối với địa hình đơn giản (đồng bằng, ít địa vật che khuất như nhà cao tầng, vật kiến trúc), khả năng chọn và đo điểm dễ dàng, độ chính xác có thể đạt mức $0.5 \mathrm{~m}$ với giãn cách khống chế 10-13km theo dải bay (mật độ trung bình khoảng $250-280 \mathrm{~km}^{2} /$ điểm). Đối với địa hình đồi núi, ven biển khả năng chọn và đo điểm khó khăn, chỉ tiêu độ chính xác từ 0.8-1,0m với mật độ điểm khống chế 10$15 \mathrm{~km}$ theo dải bay (mật độ trung bình khoảng $280 \mathrm{~km}^{2}-300 \mathrm{~km}^{2} /$ điểm).

Đối với mỗi hệ thống thiết bị bay chụp và xử lý ảnh, các thông số kỹ thuật về tăng dày ảnh hàng không kỹ thuật số được thiết lập theo tiêu chuẩn kỹ thuật tương ứng với các mức chỉ tiêu độ chính xác được thiết lập với các mẫu dữ liệu địa lý đặc trưng cho các dạng địa hình dựa trên kết quả các phép đo độ chính xác trực tiếp. Khi đó, dữ liệu địa lý được công nhận hợp chuẩn theo quy định của pháp luật về tiêu chuẩn và quy chuẩn kỹ thuật.

Chỉ tiêu độ chính xác tăng dày khống chế ảnh thể hiện bằng giá trị sai số trung phương sau bình sai khối tam giác ảnh không gian được tính toán với đồ hình các điểm khống chế và với đồ hình các điểm kiểm tra. Trong đó, đồ hình điểm khống chế bao gồm một số lượng rất lớn các điểm tâm chụp được xác định bởi công nghệ GNSS/IMU, giá trị sai số trung phương nhiều khi chưa phản ánh được độ chính xác tuyệt đối của tọa độ tăng dày. Trong trường hợp này, việc đánh giá độ chính xác cần dựa trên cả sai số trung phương được tính toán với các điểm kiểm tra (không tham gia bình sai lưới). Về nguyên tắc chỉ tiêu tăng dày khống chế ảnh cần được đảm bảo để đáp ứng các chỉ tiêu độ chính xác đo vẽ địa hình, cụ thể trong bảng dưới đây. (xem bảng 3)

\section{Trong đó:}

$m_{x, y(t)}$ là sai số trung phương theo hướng $X$ hoặc hướng $Y$ của điểm tăng dày;

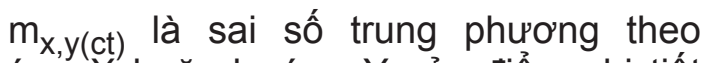
hướng $X$ hoặc hướng $Y$ của điểm chi tiết trên dữ liệu địa lý, bản đồ số, hoăc trên ảnh nắn trực giao;

$\mathrm{m}_{\mathrm{h}(\mathrm{td})}$ là sai số trung phương độ cao điểm tăng dày;

$\mathrm{m}_{\mathrm{h}(\mathrm{ct})}$ là sai số trung phương của dữ liệu độ cao mô tả địa hình mặt đất; 
$\mathrm{m}_{\mathrm{x}, \mathrm{y}(\mathrm{kc})}$ là sai số trung phương theo hướng $X$ hoặc hướng $Y$ của điểm khống chế ảnh ngoại nghiệp;

$\mathrm{m}_{\mathrm{h}(\mathrm{kc})}$ là sai số trung phương xác định độ cao của điểm khống chế ảnh ngoại nghiệp;

Sau đây là quan hệ giữa chỉ tiêu độ chính xác mặt phẳng, độ cao với độ chính xác tăng dày. (xem bảng 4,5)

c) Chỉ tiêu kỹ thuật đối với đo vẽ địa hình

Chỉ tiêu kỹ thuật đối với đo vẽ địa hình được giới hạn bằng $1 / 2$ giá trị chỉ tiêu độ chính xác tuyệt đối của dữ liệu mặt phẳng, độ cao cần đạt được. Độ chính xác đo vẽ địa hình phụ thuộc vào mức độ phức tạp của địa hình, chỉ tiêu thu nhận dữ liệu, chất lượng hình ảnh và tiêu chuẩn hóa trong nhận dạng và đo vẽ đối tượng mặt phẳng, độ cao. Chỉ tiêu kỹ thuật áp dụng trong đo vẽ bao gồm:
- Tỷ lệ mô hình hoặc tỷ lệ thu phóng hình ảnh để đo vẽ bắt buộc được lựa chọn và áp dụng như một thông số gắn với kết quả đo vẽ và sử dụng trong quá trình kiểm tra độ chính xác. Tỷ lệ thu phóng được đặt sao cho số đọc tọa độ của các trị đo lặp không vượt quá $1 / 3$ giá trị pixcel.

- Đối với phương pháp đo vẽ lập thể, ngoài tỷ lệ mô hình, mức chi tiết của độ cao đối tượng cần đo vẽ mô tả địa hình được xác định bằng $1 / 2$ mức chỉ tiêu độ chính xác độ cao $\left(\mathrm{m}_{\mathrm{hct}}\right)$ tương ứng với loại chỉ tiêu độ chính xác cần đạt được.

- Đối với đo vẽ thu nhận dữ liệu địa lý, mức độ chi tiết $m_{x y c t}$ không vượt quá $1 / 2$ giá trị chỉ tiêu độ chînh xác cần đạt được.

Kết quả đánh giá độ chính xác đo vẽ được thực hiện bằng phương pháp đo điểm kiểm tra, lấy mẫu dữ liệu. Tập trị đo được sử dụng để tính toán số chênh tọa độ đo trùng có thể có được từ kết quả tăng dày đã được

Bảng 3:

\begin{tabular}{|c|c|c|c|c|}
\hline \multirow{2}{*}{ ……u c.... uny } & \multicolumn{2}{|c|}{ Độ chính xác tăng dày } & \multicolumn{2}{c|}{ Độ chính xác khống chế } \\
\cline { 2 - 5 } & Mặt phẳng $\mathrm{m}_{\mathrm{x}, \mathrm{y}(\mathrm{t} \mathrm{d})}$ & Độ cao $\mathrm{m}_{\mathrm{h}(\mathrm{t} \mathrm{d})}$ & Mặt phẳng $\mathrm{m}_{\mathrm{x}, \mathrm{y}(\mathrm{kc})}$ & Độ cao $\mathrm{m}_{\mathrm{h}(\mathrm{kc})}$ \\
\hline Về mặt phẳng: $\mathrm{m}_{\mathrm{x}, \mathrm{y}(\mathrm{ct})}$ & $1 / 2 \mathrm{~m}_{\mathrm{x}, \mathrm{y}(\mathrm{ct})}$ & $\mathrm{m}_{\mathrm{x}, \mathrm{y}(\mathrm{ct})}$ & $1 / 4 \mathrm{~m}_{\mathrm{x}, \mathrm{y}(\mathrm{ct})}$ & $1 / 2 \mathrm{~m}_{\mathrm{x}, \mathrm{y}(\mathrm{ct})}$ \\
\hline Về độ cao $\mathrm{mh}(\mathrm{ct})$ & $1 / 2 \mathrm{~m}_{\mathrm{x}, \mathrm{y}(\mathrm{ct})}$ & $1 / 2 \mathrm{~m}_{\mathrm{h}(\mathrm{ct})}$ & $1 / 4 \mathrm{~m}_{\mathrm{x}, \mathrm{y}(\mathrm{ct})}$ & $1 / 2 \mathrm{~m}_{\mathrm{h}(\mathrm{ct})}$ \\
\hline
\end{tabular}

Bảng 4: Chỉ tiêu độ chính xác tăng dày mặt phẳng

\begin{tabular}{|c|c|c|c|c|c|}
\hline $\begin{array}{c}\text { Chỉ tiêu } \\
\text { ĐCX mặt } \\
\text { phẳng } \\
\text { Rms }\left(m_{x y}\right)\end{array}$ & $\begin{array}{c}\text { Độ } \\
\text { phẩn } \\
\text { giải } \\
\text { (GSD) }\end{array}$ & $\begin{array}{c}\text { Độ cao } \\
\mathrm{BC} \mathrm{H}, \\
\text { tương } \\
\text { ứng } \\
\text { với } \mathrm{k}\end{array}$ & $\begin{array}{l}\text { Chỉ tiêu } \\
\text { ĐCX } \\
\text { tăng dày } \\
\text { mặt } \\
\text { phẳng } \mathrm{m}_{\mathrm{td}}\end{array}$ & $\begin{array}{c}\text { Tương } \\
\text { ứng với } \\
\text { tỷ lệ bản } \\
\text { đồ }\end{array}$ & $\begin{array}{l}\text { Loại } \\
\text { ĐCX } \\
\left(m_{x, y}\right)\end{array}$ \\
\hline$(\mathrm{cm})$ & $(\mathrm{cm})$ & (m) & $(\mathrm{cm})$ & & \\
\hline 0.25 & 0.08 & 979 & 0.125 & $1: 1.000$ & $I$ \\
\hline 0.5 & 0.17 & 1.958 & & & II \\
\hline 0.75 & 0.25 & 2.938 & & & III \\
\hline 0.5 & 0.17 & 1.958 & 0.25 & $1: 2.000$ & $I$ \\
\hline 1 & 0.33 & 3.917 & & & II \\
\hline 1.5 & 0.50 & 5.875 & & & III \\
\hline 1.25 & 0.42 & 4.896 & 0.625 & $1: 5.000$ & $I$ \\
\hline 2.5 & & & & & II \\
\hline 3.75 & & & & & III \\
\hline 2.5 & & & & $1: 10.000$ & $I$ \\
\hline 5 & & & & & II \\
\hline 7.5 & & & & & III \\
\hline
\end{tabular}

Bảng 5: Chỉ tiêu độ chính xác tăng dày cả mặt phẳng, độ cao (đơn vị m)

\begin{tabular}{|c|c|c|c|c|c|}
\hline $\begin{array}{c}\text { Chỉ tiêu } \\
\text { độ chính } \\
\text { xác độ } \\
\text { cao }\end{array}$ & $\begin{array}{c}\text { Độ } \\
\text { cao } \\
\text { bay } \\
\text { chụp }\end{array}$ & $\begin{array}{c}\text { Độ } \\
\text { phân } \\
\text { giải } \\
\text { mắt } \\
\text { đất }\end{array}$ & $\begin{array}{c}\text { Mâu sô tỳ } \\
\text { lệnh } \\
\text { ảnh } \\
\text { với } \\
\mathrm{p}=60 \%\end{array}$ & $\begin{array}{c}\text { Chỉ tiêu độ } \\
\text { chính xác } \\
\text { tăng dày độ } \\
\text { cao }\end{array}$ & $\begin{array}{c}\text { Tương ứng } \\
\text { với khoảng } \\
\text { cao đêu cơ } \\
\text { bản }\end{array}$ \\
\hline $\mathrm{m}_{\mathrm{h}}$ & $\mathrm{H}$ & $\mathrm{GSD}$ & $\mathrm{m}_{\mathrm{a}}$ & $\mathrm{m}_{\mathrm{td}}=\left(\mathrm{m}_{\mathrm{h}}\right) / 2$ & $\mathrm{~h}(\mathrm{~m})$ \\
\hline 0.13 & 566 & 0.05 & 8,021 & 0.063 & 0.5 \\
\hline 0.25 & 1131 & 0.10 & 16,043 & 0.125 & 1 \\
\hline 0.30 & 1357 & 0.12 & 19,251 & 1.150 & - \\
\hline 0.40 & 1810 & 0.15 & 25,668 & 0.200 & - \\
\hline 0.50 & 2262 & 0.19 & 32,085 & 0.250 & - \\
\hline 0.60 & 2714 & 0.23 & 38,502 & 0.300 & - \\
\hline 0.83 & 3755 & 0.32 & 53,261 & 0.415 & 2.5 \\
\hline 1.00 & 4524 & 0.39 & 64,170 & 0.500 & - \\
\hline 1.66 & - & - & - & 0.830 & 5 \\
\hline 3.33 & - & - & - & - & - \\
\hline 6.66 & - & - & - & - & - \\
\hline
\end{tabular}


sử dụng để đo vẽ hoặc nắn ảnh trực giao.

\subsection{Thống kê, báo cáo độ chính xác}

Thống kê, báo cáo độ chính xác đạt được của sản phẩm dữ liệu địa lý dựa trên kết quả thực hiện các phép đo độ chính xác trực tiếp trên tập dữ liệu được lấy mẫu theo quy định. Theo đó, việc áp dụng chỉ tiêu độ chính xác được công bố với độ tin cậy $95 \%$ giá trị sai số của tập dữ liệu được phân bố chuẩn và sai số hệ thống đều đã được loại bỏ.

Tham chiếu với thực tế công tác quản lý chất lượng về độ chính xác hiện nay, ngoài việc áp dụng các chỉ tiêu đánh giá độ chính xác trực tiếp, kết luận về đạt mức chỉ tiêu độ chính xác có thể được chấp nhận hợp chuẩn, hợp quy khi áp dụng các quy chuẩn, tiêu chuẩn kỹ thuật liên quan đến độ chính xác đã được các cơ quan thẩm quyền công bố theo quy định của Luật tiêu chuẩn và quy chuẩn kỹ thuật (phương pháp gián tiếp). Bao gồm: các tiêu chuẩn về quá trình để tạo ra dữ liệu và các chỉ tiêu kỹ thuật áp dụng đối với các công đoạn: Thu nhận dữ liệu ảnh và các nguyên tố định hướng ngoài; Bình sai lưới TGAKG; Đo vẽ địa hình và gia công đóng gói các sản phẩm dữ liệu địa lý có liên quan đến độ chính xác.

Kết quả báo cáo về độ chính xác được ghi nhận vào siêu dữ liệu với xác nhận của chủ đầu tư là căn cứ để nghiệm thu và thanh quyết toán theo đơn giá, định mức phù hợp.

\section{Kết luận}

Bộ chỉ tiêu về độ chính xác đo đạc địa hình trên nền công nghệ đo ảnh kỹ thuật số được áp dụng phù hợp với các yêu cầu thực hiện phép đo chất lượng dữ liệu địa lý theo Quy chuẩn quốc gia, mã số QCVN42: 2012/BTNMT và các văn bản kỹ thuật liên quan.

Các chỉ tiêu kỹ thuật đánh giá chất lương theo phương pháp gián tiếp được áp dụng để xây dựng các tiêu chuẩn kỹ thuật đối với các hạng mục công trình, sản phẩm dữ liệu địa lý thay thế cho các quy định kỹ thuật hiện hành đã không còn phù hợp. Trong đó, đối tượng áp dụng tiêu chuẩn là các sản phẩm thuộc danh mục đã có định mức và quy trình để tạo ra các sản phẩm đó. $\mathrm{O}$

\section{Tài liệu tham khảo}

[1]. Thông tư số 02 /2012/TT-BTNMT ngày 19 tháng 03 năm 2012 quy định Quy chuẩn kỹ thuật quốc gia về chuẩn thông tin địa lý cơ sở, ban hành theo thể thức Quy chuẩn quốc gia, mã số QCVN42: 2012/BTNMT.

[2]. Geospatial Positioning Accuracy Standards, Part 3: National Standard for Spatial Data Accuracy, Federal Geographic Data Committee.

[3]. The American Society for Photogrammetry and Remote Sensing Approval by the ASPRS Professional Practicing Division, March, 1990, ASPRS American Society of Photogrammetry and Remote Sensing. $O$

\section{Summary}

\section{Innovate criteria for measuring the accuracy of terrain measurements based on digital imaging technology}

Nguyen Thi Bich Ngoc, Department of Surveying, Mapping and Geoinformation of Vietnam

Dao Thi Nga, Vietnam Natural Resources and Environment Corporation

In recent years, technologies in Mapping and capturing Geographic data have changed, many new technologies haved appled in Viet Nam. Meanwhile, in some specification documents, the evaluation of product quality still apply under the old regulations. This paper presents the research results in the renovation of the criteria for assessing the accurancy of topographic surveying based on digital photogrammetric technology. $O$ 